\title{
Model Agnostic Time Series Analysis via Matrix Estimation
}

\author{
Anish Agarwal \\ MIT \\ anish90@mit.edu \\ Devavrat Shah \\ MIT \\ devavrat@mit.edu
}

\author{
Muhammad Jehangir Amjad \\ MIT \\ mamjad@mit.edu \\ Dennis Shen \\ MIT \\ deshen@mit.edu
}

\begin{abstract}
We propose an algorithm to impute and forecast a time series by transforming the observed time series into a matrix, utilizing matrix estimation to recover missing values and de-noise observed entries, and performing linear regression to make predictions. At the core of our analysis is a representation result, which states that for a large class of models, the transformed time series matrix is (approximately) low-rank. In effect, this generalizes the widely used Singular Spectrum Analysis (SSA) in the time series literature, and allows us to establish a rigorous link between time series analysis and matrix estimation. The key to establishing this link is constructing a Page matrix with non-overlapping entries rather than a Hankel matrix as is commonly done in the literature (e.g., SSA). This particular matrix structure allows us to provide finite sample analysis for imputation and prediction, and prove the asymptotic consistency of our method. Another salient feature of our algorithm is that it is model agnostic with respect to both the underlying time dynamics and the noise distribution in the observations. The noise agnostic property of our approach allows us to recover the latent states when only given access to noisy and partial observations a la a Hidden Markov Model; e.g., recovering the time-varying parameter of a Poisson process without knowing that the underlying process is Poisson. Furthermore, since our forecasting algorithm requires regression with noisy features, our approach suggests a matrix estimation based method-coupled with a novel, non-standard matrix estimation error metric-to solve the error-in-variable regression problem, which could be of interest in its own right. Through synthetic and real-world datasets, we demonstrate that our algorithm outperforms standard software packages (including R libraries) in the presence of missing data as well as high levels of noise.
\end{abstract}

\section{KEYWORDS}

Time Series Analysis, Matrix Estimation, Hidden Markov Models, Linear-Time-Invariant Systems

\section{ACM Reference Format:}

Anish Agarwal, Muhammad Jehangir Amjad, Devavrat Shah, and Dennis Shen. 2019. Model Agnostic Time Series Analysis via Matrix Estimation. In ACM SIGMETRICS / International Conference on Measurement and Modeling

Permission to make digital or hard copies of part or all of this work for personal or classroom use is granted without fee provided that copies are not made or distributed for profit or commercial advantage and that copies bear this notice and the full citation on the first page. Copyrights for third-party components of this work must be honored. For all other uses, contact the owner/author(s).

SIGMETRICS '19 Abstracts, fune 24-28, 2019, Phoenix, AZ, USA

(c) 2019 Copyright held by the owner/author(s).

ACM ISBN 978-1-4503-6678-6/19/06.

https://doi.org/10.1145/3309697.3331479 of Computer Systems (SIGMETRICS '19 Abstracts), June 24-28, 2019, Phoenix, AZ, USA. ACM, New York, NY, USA, 2 pages. https://doi.org/10.1145/3309697. 3331479

\section{INTRODUCTION}

Time series data is of enormous interest across all domains of life: from health sciences and weather forecasts to retail and finance, time dependent data is ubiquitous. Despite the diversity of applications, time series problems are commonly confronted by the same two pervasive obstacles: interpolation and extrapolation in the presence of noisy and/or missing data. Specifically, we consider a discrete-time setting with $t \in \mathbb{Z}$ representing the time index and $f: \mathbb{Z} \rightarrow \mathbb{R}$ representing the latent discrete-time time series of interest. For each $t \in[T]:=\{1, \ldots, T\}$ and with probability $p \in(0,1]$, we observe the random variable $X(t)$ such that $\mathbb{E}[X(t)]=f(t)$. While the underlying mean signal $f$ is of course strongly correlated, we assume the per-step noise is independent across $t$ and has uniformly bounded variance. Under this setting, we have two objectives: (1) interpolation, i.e., estimate $f(t)$ for all $t \in[T]$; (2) extrapolation, i.e., forecast $f(t)$ for $t>T$. Our interest is in designing a generic method for interpolation and extrapolation that is applicable to a large model class while being agnostic to the time dynamics and noise distribution.

We develop an algorithm based on matrix estimation, a topic which has received widespread attention, especially with the advent of large datasets. In the matrix estimation setting, there is a "parameter" matrix $\boldsymbol{M}$ of interest, and we observe a sparse, corrupted signal matrix $X$ where $\mathbb{E}[X]=M$. The aim then is to recover the entries of $\boldsymbol{M}$ from noisy and partial observations given in $\boldsymbol{X}$. For our purposes, the attractiveness of matrix estimation derives from the property that these methods are fairly model agnostic in terms of the structure of $\boldsymbol{M}$ and distribution of $\boldsymbol{X}$ given $\boldsymbol{M}$. We utilize this key property to develop a model and noise agnostic time series imputation and prediction algorithm.

\subsection{Overview of contributions}

Time series as a matrix. We transform the time series of observations $X(t)$ for $t \in[T]$ into what is known as the Page matrix by placing contiguous segments of size $L>1$ (an algorithmic hyperparameter) of the time series into non-overlapping columns.

As the key contribution, we establish that-in expectation-this generated matrix is either exactly or approximately low-rank for a large class of models $f$. Specifically, $f$ can be from:

Linear Recurrent Formulae (LRF): $f(t)=\sum_{g=1}^{G} \alpha_{g} f(t-g)$. 
Compact Support: $f(t)=g(\varphi(t))$ where $\varphi: \mathbb{Z} \rightarrow\left[-C_{1}, C_{1}\right]$ has the form $\varphi(t+s)=\sum_{l=1}^{G} \alpha_{l} a_{l}(t) b_{l}(s)$ with $\alpha_{l} \in\left[-C_{2}, C_{2}\right], a_{l}: \mathbb{Z} \rightarrow$ $[0,1], b_{l}: \mathbb{Z} \rightarrow[0,1]$ for some $C_{1}, C_{2}>0$; and $g:\left[-C_{1}, C_{1}\right] \rightarrow \mathbb{R}$ is $\mathcal{L}$-Lipschitz ${ }^{1}$.

Sublinear: $f(t)=g(t)$ where $g: \mathbb{R} \rightarrow \mathbb{R}$ and $\left|\frac{d g(s)}{d s}\right| \leq C s^{-\alpha}$ for some $\alpha, C>0$, and $\forall s \in \mathbb{R}$.

Imputation: Over the past decade, the matrix estimation community has developed a plethora of methods to recover an exact or approximately low-rank matrix from its noisy, partial observations in a noise and model agnostic manner. Therefore, by applying such a matrix estimation method to this transformed matrix, we can recover the underlying mean matrix (and thus $f(t)$ for $t \in[T]$ ) accurately. In other words, we can interpolate and de-noise the original corrupted and incomplete time series without any knowledge of its time dynamics or noise distribution. We provide finite-sample analyses for this method and establish the consistency property of our algorithm, as long as the underlying $f$ and the matrix estimation method satisfy some natural properties. We show that any additive mixture of the three function classes listed above satisfies these properties. Effectively, we establish a statistical reduction between time series imputation and matrix estimation. Our key contribution with regards to imputation lies in establishing that a large class of time series models (any additive mixture of the three listed above) can be effectively denoised.

Forecasting: It is clear that for LRF, the last row of the mean transformed matrix can be expressed as a linear combination of the other rows. An important representation result of the present paper, which generalizes this notion, is that an approximate LRF relationship holds for the other two model classes. Therefore, we can forecast $f(t)$, say for $t=T+1$, as follows: apply matrix estimation to the transformed data matrix as done in imputation; then, linearly regress the last row with respect to the other rows in the matrix; finally, compute the inner product of the learnt regression vector with the vector containing the previous $L-1$ values that were estimated via the matrix estimation method. We show that the mean-squared error of our predictions decays to zero provided the matrix estimation method satisfy some natural properties. Similar to the case of imputation, establishing that these properties hold for the three function classes is novel.

Noisy regression. Our proposed forecasting algorithm performs regression with noisy and incomplete features i.e., error-in-variable regression. There has been exciting progress to understand this problem, especially in the high-dimensional setting. Our algorithm offers an alternate solution through the lens of matrix estimation: first, utilize matrix estimation to de-noise and impute the feature observations, and then perform least squares with the pre-processed feature matrix. We demonstrate that if the true, underlying feature matrix is (approximately) low-rank, then our algorithm provides a consistent estimator to the true signal. Our analysis further suggests the usage of a non-standard error metric, the max row sum error.

Class of applicable models. As aforementioned, our algorithm enjoys strong performance guarantees provided the underlying

\footnotetext{
${ }^{1}$ It can be verified that if $\varphi$ is an LRF satisfying $\varphi(t)=\sum_{h=1}^{H} \gamma_{h} \varphi(t-h)$, then it satisfies the form $\varphi(t+s)=\sum_{g=1}^{G} \alpha_{g} a_{g}(t) b_{g}(s)$ for $G=H$ with appropriately defined constants $\alpha_{g}$, functions $a_{g}, b_{g}$
}

mean matrix induced by the time series $f$ satisfies certain structural properties. We argue that a broad class of standard time series models meets the requirements of the function classes listed above.

LRFs include the following important family of time series: a finite sum of products of exponentials $(\exp \{\alpha t\})$, harmonics $(\cos (2 \pi \omega t+\phi))$, and finite degree polynomials $\left(P_{m}(t)\right)$, i.e.,

$$
f(t)=\sum_{g=1}^{G} \exp \left\{\alpha_{g} t\right\} \cos \left(2 \pi \omega_{g} t+\phi_{g}\right) P_{m_{g}}(t) .
$$

Further, since stationary processes and $L_{2}$ integrable functions are well approximated by a finite summation of harmonics (i.e., sin and cos), LRFs encompass a vitally important family of models. For this model, we show the required structural properties of the induced time series matrix for imputation and prediction are satisfied.

However, there are many important time series models that do not admit a finite order LRF representation. A few toy examples include $\cos (\sin (t)), \exp \left\{\sin ^{2}(t)\right\}, \log t, \sqrt{t}$. Time series models with compact support, on the other hand, include models composed of a finite summation of periodic functions (e.g., $\left.\cos (\sin (t)), \exp \left\{\sin ^{2}(t)\right\}\right)$. Utilizing our low-rank representation result, we establish that models with compact support possess the desired structural properties. We further demonstrate that sublinear functions, which include models that are composed of a finite summation of non (super-) linear functions (e.g., $\log t, \sqrt{t}$ ), also possess the necessary structural properties. Importantly, we argue that the finite mixture of the above processes satisfy the necessary structural properties.

Recovering the hidden state. Our algorithm, being noise and time-dynamics agnostic, makes it relevant to recover the hidden state from its noisy, partial observations as in a Hidden Markov-like Model. For example, imagine having access to partial observations of a time-varying truncated Poisson process ${ }^{2}$ without knowledge that the process is Poisson. By applying our imputation algorithm, we can recover time-varying parameters of this process accurately and, thus, the hidden states. If we were to apply an ExpectationMaximization (EM) like algorithm, it would require knowledge of the underlying model being Poisson; moreover, theoretical guarantees are not clear for such an approach.

Sample complexity. Given the model agnostic nature of our algorithm, it is expected that sample complexity for a specific model class will be worse than model aware optimal algorithms. Interestingly, our finite sample analysis suggests that for the model classes stated above, the performance loss incurred is minor.

Experiments. Using synthetic and real-world datasets, our experiments establish that our method outperforms existing standard software packages (including R) for the tasks of interpolation and extrapolation in the presence of noisy and missing observations. When the data is generated synthetically, we "help" the existing software package by choosing the correct parametric model and algorithm while our algorithm remains oblivious to the underlying model; despite this disadvantage, our algorithm continues to outperform the standard packages with missing data.

Further, our empirical studies demonstrate that our imputation algorithm accurately recovers the hidden state for Hidden Markovlike Models, verifying our theoretical imputation guarantees.

${ }^{2}$ Let $C$ denote a positive, bounded constant, and $X$ a Poisson random variable. We define the truncated Poisson random variable $Y$ as $Y=\min \{X, C\}$. 